\title{
Community violence and urban childhood asthma: a multilevel analysis
}

\author{
M.J. Sternthal*, H-J. Jun\#, F. Earls ${ }^{\tau,+}$ and R.J. Wright", ${ }^{\#, \S}$
}

ABSTRACT: We examined the association between community violence exposure and childhood asthma risk in a multilevel, multimethod, longitudinal study controlling for individual- and neighbourhood-level confounders and pathway variables.

Analyses included 2,071 children aged 0-9 yrs at enrolment from the Project on Human Development in Chicago Neighborhoods. Multilevel logistic regression models estimated the likelihood of asthma, controlling for individual-level (child's age, sex, race/ethnicity, maternal asthma, socioeconomic status and family violence in the home) and neighbourhood-level confounders (concentrated disadvantage, collective efficacy and social disorder), and pathway variables (maternal smoking, breastfeeding).

In adjusted analyses, medium (OR 1.60, 95\% $\mathrm{Cl} 1.17-2.19)$ and high levels (OR 1.56, 95\% $\mathrm{Cl}$ 1.12-2.18) of community violence were associated with increased asthma risk, relative to low levels. The increased asthma risk remained for African Americans when models included community violence and all other individual-level covariates, but attenuated to borderline nonsignificance when further adjusting for collective efficacy.

Community violence is associated with asthma risk when controlling for individual- and neighborhood-level confounders. Neither community violence, nor the other individual-level factors, fully accounted for the excess asthma burden among African Americans. These data suggest that public health interventions outside the biomedical model may be needed to reduce asthma in disadvantaged populations.

KEYWORDS: Asthma, collective efficacy, community violence, multilevel analyses, neighbourhood disadvantage, social disorder

$\mathrm{n}$ the USA, those living in impoverished urban neighbourhoods have the highest asthma rates and associated morbidity relative to their higher socioeconomic status (SES) and nonurban counterparts [1]. Such disparities are not adequately explained by physical environmental factors [2]. The recognised importance of the social environment in child health, coupled with knowledge of the mechanisms linking psychological stress and asthma [2, 3] indicate that children may be raised in social contexts potentially as detrimental to their development and health as physical toxins [4].

Chronic community violence has been identified as a prevalent and extreme stressor confronting many urban poor communities $[5,6]$. In one inner-city cohort in Chicago, IL, USA, $42 \%$ of children aged 7-13 yrs had seen someone shot and $37 \%$ had seen someone stabbed [6].

Moreover, community violence contributes significantly to urban childhood morbidity [5]. Witnessing or being a victim of community violence adversely affects a child's cognitive, emotional and social functioning [7, 8]. Research links community violence exposure to alterations in the physiological stress response, including increased blood pressure and heart rate, and disrupted cortisol expression [8, 9]. Such biobehavioural and physiological sequelae may lead to broader health effects. Accumulating evidence suggests that community violence may contribute to the burden of asthma in urban populations [5]. Increased exposure is associated with more symptom days [10], higher hospitalisation rates [11], increased asthma prevalence among children in communities with both elevated crime/ violence and other environmental hazards (i.e. ambient air pollutants) [12], and increased risk of wheezing at 2-3 yrs of age [13].

While evidence suggests that community violence may contribute to asthma expression, there are methodological limitations. First, studies have not accounted for the co-occurrence of other childhood adversities that may also contribute to

\section{AFFILIATIONS}

*Dept of Environmental and Occupational Medicine and Epidemiology,

${ }^{+}$Dept of Society, Human Development and Health, ${ }^{\S}$ Dept of Environmental Health, Harvard School of Public Health, \#Channing Laboratory, Brigham and Women's Hospital, and 'Dept of Global Health and Social Medicine, Harvard Medical School, Boston, MA, USA.

CORRESPONDENCE

R.J. Wright

Channing Laboratory

Havard Medical School

181 Longwood Ave

Boston

MA 02115

USA

E-mail: msternth@hsph.harvard.edu

Received:

Jan 062010

Accepted after revision:

April 132010

First published online:

April 222010 
psychological stress and asthma (e.g. interfamilial violence) [14]. Secondly, none of the existing studies consider neighbourhood structural measures. This is problematic because community violence clusters in areas characterised by other community-level social stressors that also tax individuals living in these neighbourhoods. These include constructs of neighbourhood disadvantage, social disorder and low collective efficacy $[5,15]$. For example, the real or perceived threat of crime, and the broader construct of social disorder, fosters a distrust of others, disrupted collective efficacy and social isolation [15], which, in turn have been linked to asthma outcomes in low-income families [16]. Moreover, those experiencing greater community violence may also be exposed to physical toxins linked to asthma expression (air pollution and indoor allergens) [17]. Thirdly, studies to date have been cross-sectional or retrospective, preventing demonstration of a temporal relationship between community violence exposure and asthma development.

We address some of these limitations by examining whether higher level exposure to community violence is associated with an increased risk of asthma in a prospective study of urban children, after controlling for individual-level sociodemographics, behaviour (smoking or breastfeeding), family violence and neighbourhood-level measures of disadvantage, social disorder and collective efficacy.

\section{METHODS}

\section{Study design and analytic sample}

Data are from the Project on Human Development in Chicago Neighborhoods (PHDCN), a multilevel, multimethod, longitudinal study of contextual factors and individual development [18]. Figure 1 illustrates the data collection schema and current analytic sample. Briefly, 343 Chicago neighbourhood clusters (NCs) were identified using 1990 US Census data. In 1995, an interview-administered community survey (CS) was conducted with a representative sample of adults within each of the NCs $(n=8,782)$ to derive neighbourhood-level constructs (i.e. social disorder and collective efficacy detailed below) independent of the sample from which individual-level data were ascertained.

For the longitudinal study, a stratified probability sample of 80 neighbourhoods was selected from the 343 NCs. Conceived using an accelerated cohort design, English- and Spanishspeaking households were screened for eligible children within 6 months of seven target cohort ages (0 (in utero to age 6 months), 3, 6, 9, 12, 15 and 18 yrs). Within each household, all eligible children were invited to participate, along with the primary caregiver for those $<18$ yrs of age. Participants were enrolled in 1995 and followed in three waves of data collection: wave 1 (1994-1997), wave 2 (1997-1999) and wave 3 (2000-2001). The institutional review board of the

Individual-level variables

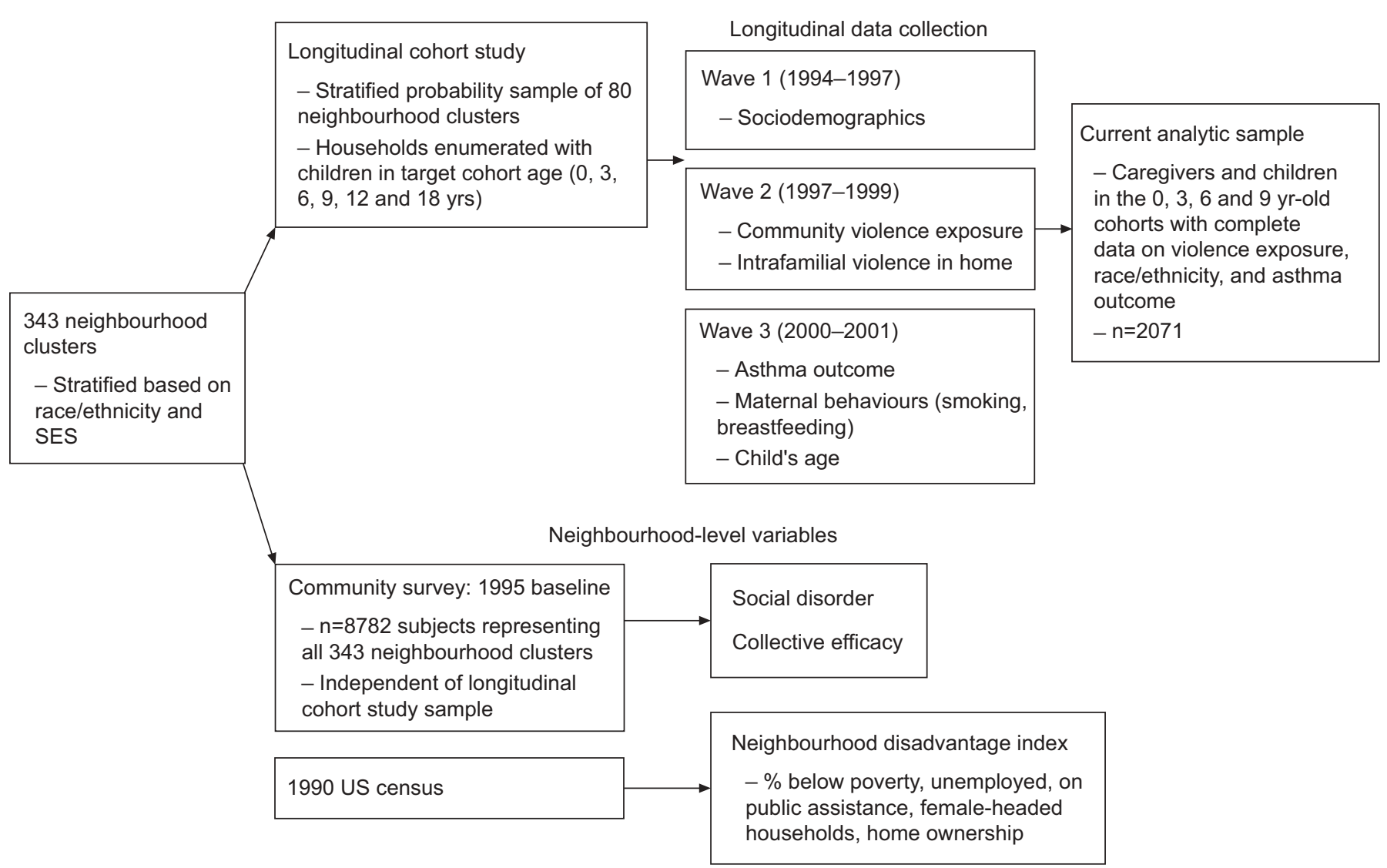

FIGURE 1. Project on Human Development in Chicago Neighbourhoods: data collection schema and current analytic sample. SES: socioeconomic status. 
Harvard School of Public Health (Boston, MA, USA) approved secondary analyses using the public database.

\section{Community violence exposure}

At wave 2, primary caregivers completed the My Exposure to Violence (ETV) survey, a structured, interviewer-administered measure ascertaining the child's lifetime exposure to violent events in their neighbourhood $[19,20]$. Internal consistency, test-retest reliability and validity have been established, including the Spanish translation [21]. For endorsed items, follow-up questions inquire about factors known to influence the impact of violence, including familiarity with the perpetrator or victim, events occurring more than once and whether events occurred outside the home. For the main exposure, we considered only events witnessed and/or experienced outside the home (i.e. neighbourhood violence) in the child's lifetime prior to asthma diagnosis.

Rasch modelling was used to summarise the multi-item responses (table 1) into a continuous ETV composite variable [7]. Models were fitted using logistic, nonlinear, mixed models (NLMIXED) in SAS 9.0 (SAS Institute, Cary, NC, USA) specifying a binary distribution and a random effect, defined to have mean of 0 and a variance estimated by the modelfitting process. Higher scores indicate greater exposure. ETV

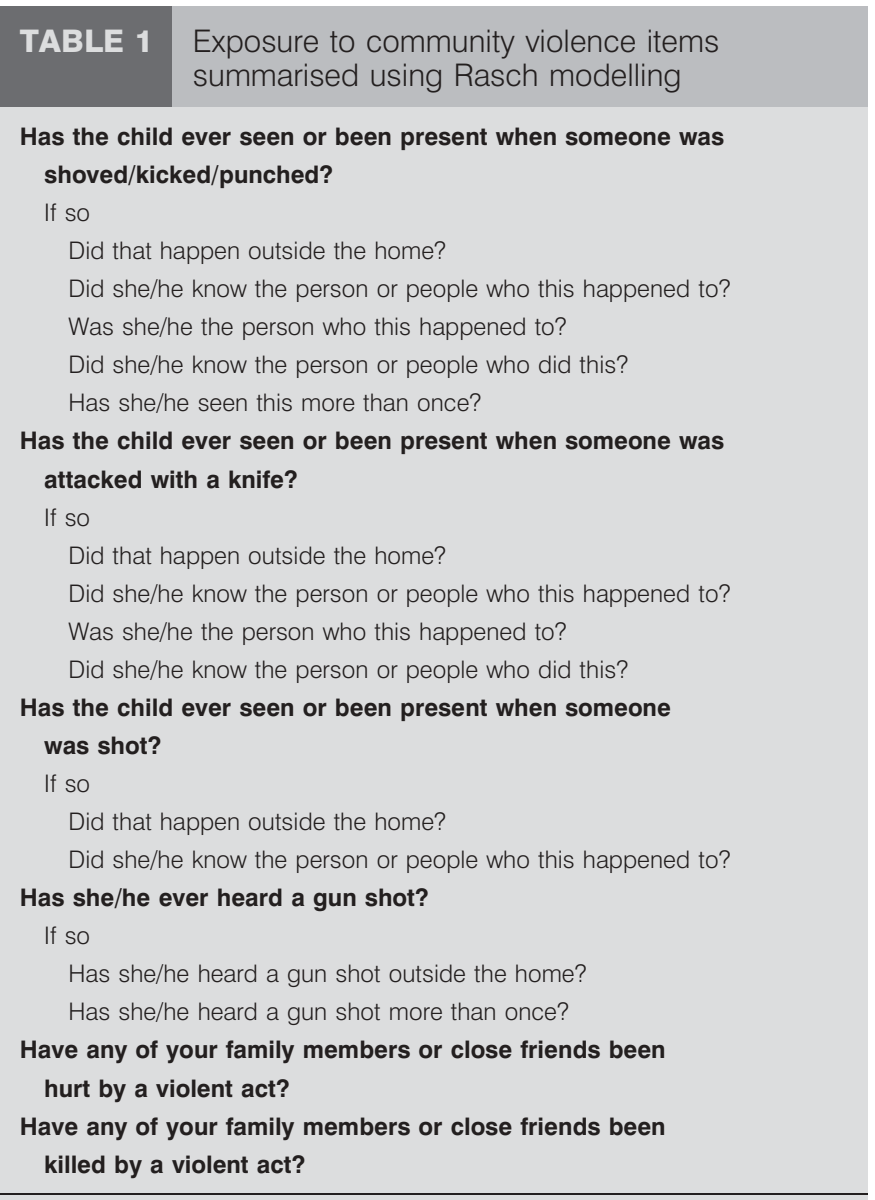

All responses were either "yes" or "no" was examined as a continuous indicator and divided into tertiles to test for an exposure-response relationship.

\section{Asthma outcome}

In wave 3 , standardised questions adapted from the American Thoracic Society Division of Lung Diseases (ATS-DLD-78) questionnaire [22] were administered to the primary caregivers of children from the age cohorts who were 0-9 yrs of age at enrolment. The analytic sample included everyone in these age groups who completed the survey $(n=2,228)$ (response rate $75.8 \%$ ), excluding 152 children missing information on community violence exposure and five missing information on race/ethnicity, to give a total of $n=2,071$. The primary outcome was defined as having ever been diagnosed with asthma or having ever taken prescription asthma medicine.

We considered alternative definitions in sensitivity analyses. Because having a current diagnosis of wheeze is associated with more objective measures, including airway responsiveness [23], we alternatively examined current asthma, which was defined as having both a diagnosis and reporting symptoms or medication use indicative of asthma (i.e. wheezing or using prescription medication for wheezing) in the past year. We also considered the potential for unconfirmed possible asthma, defined as never having been diagnosed with asthma but reporting symptoms (ever wheezing) or prescription medication use for wheezing illnesses. The latter definition was considered to address concerns about underdiagnosis of asthma potentially attributable to racial or ethnic disparities in access to healthcare [24] or cultural differences in acceptability of a diagnosis of asthma [25].

\section{Covariates}

Variables previously related to community violence exposure and childhood asthma were examined as summarised in figure $2[5,11,14,26,27]$.

\section{Individual-level confounders}

Sociodemographics

Variables ascertained in wave 1 included maternal education, child's race/ethnicity and sex. Child's age at wave 3 was also used. Equivalised income was calculated by dividing household income by the square root of the number of household members [28].

\section{Maternal asthma}

Maternal asthma, ascertained at wave 3, was defined as having ever been diagnosed with asthma by a physician asthma [22].

\section{Family violence in the home}

Maternal report of the child's exposure to family violence was obtained in wave 2 using the Conflict Tactics Scale (CTS) Form R assessing the extent to which she and her partner psychologically and physically attacked one another in the past year [29]. Reliability and validity data are available, including the Spanish translation [30]. Items assessed psychological aggression (e.g. insulting/swearing and threatening violence) and physical violence (e.g. pushing or slapping; kicking, hitting, or beating; or using a weapon). Participants rated whether items occurred never, once, twice, 3-5, 6-10, 11-20, or more than 20 times as both victim and perpetrator. Summed scores were included as a three-level categorical indicator representing none (score of 0 ), 


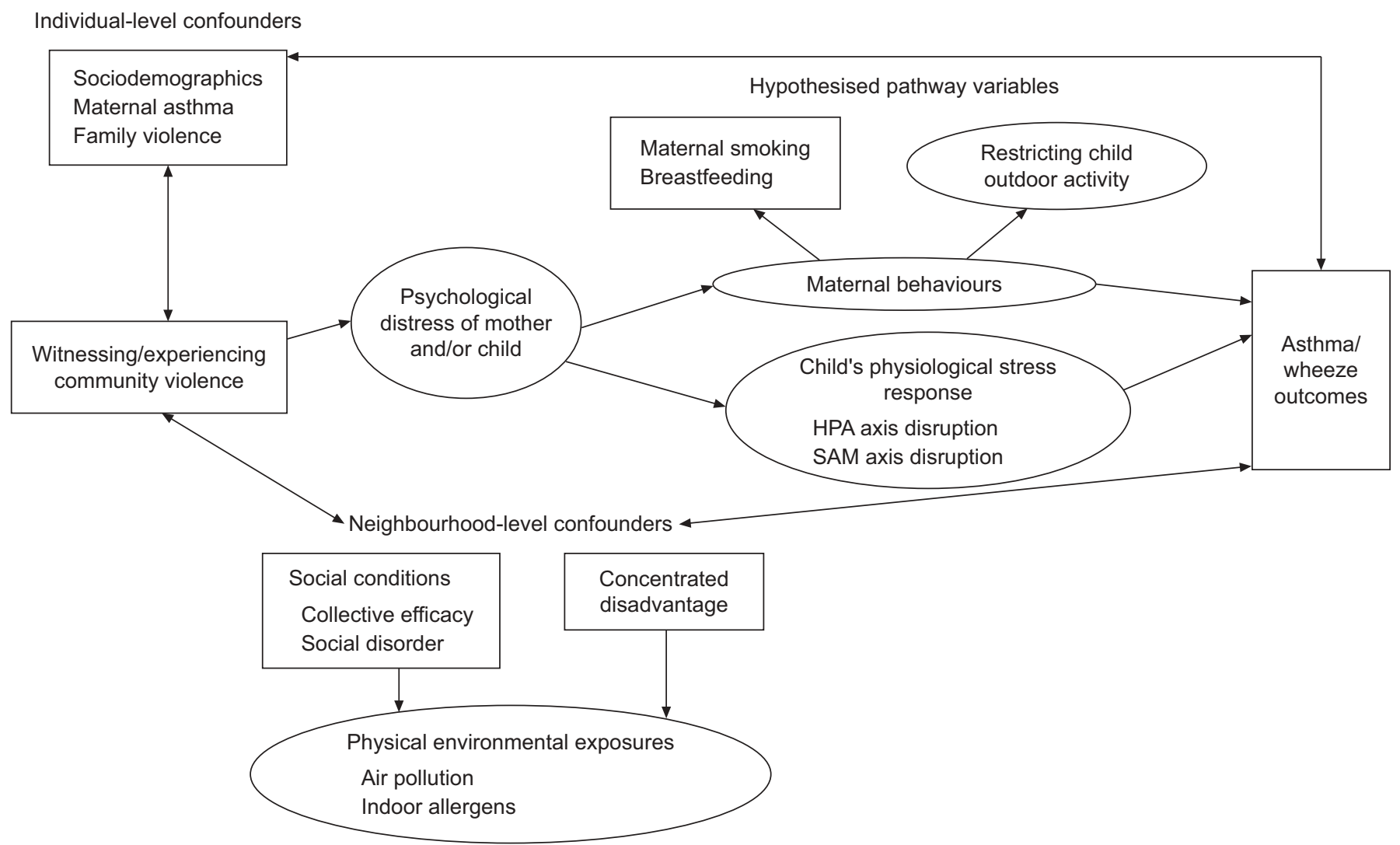

FIGURE 2. Conceptual diagram illustrating relationships among variables linking community violence and childhood asthma. Ovals represent unmeasured or latent variables and boxes represent measure variables. HPA: hypothalamic-pituitary-adrenal; SAM: sympathetic-adrenal-medullary.

low (less than the median value, range 1-25) and high (above the median value, range 26-488).

\section{Neighbourhood-level confounders Neighbourhood disadvantage}

Neighbourhood disadvantage, characterised by communitylevel stressors beyond violence (e.g. concentrated poverty, unemployment and segregation) may also be a surrogate marker of increased exposure to other environmental exposures not directly assessed in this study (e.g. ambient pollutants and indoor allergens) [17]. A disadvantage index was derived for each NC using aggregated data from the 1990 US Census (independent of the PHDCN-CS) based on an average $z$-score for: 1) percentage living below poverty, 2) percentage unemployed, 3) percentage on public assistance, 4) percentage female-headed households, 5) percentage youth, and 6) percentage African Americans [31].

\section{Social disorder}

Research links social disorder to crime and poor health [32]. Social disorder itself, or increased crime in this context (i.e. crime that subjects are aware of but have not personally experienced) may independently affect health, including asthma [11]. The three-item scale asked how much of a neighbourhood problem (ranging from 0 ("no problem") to 2 ("serious problem")) are: drinking in public, selling or using drugs, and teenagers or adults loitering and causing trouble. Items were summed, with higher scores indicating greater disorder.

\section{Collective efficacy}

Collective efficacy measures the level of trust among residents and their perceived willingness to engage in collective action [18]. Conversely, increased disorder fosters mistrust, isolation and a perception of community indifference to individuals in need [15]. Neighbourhoods with high collective efficacy often report less crime [31] and better health [33]. Collective efficacy was derived using a composite measure based on the PHDCNCS social cohesion and informal social control scales [18]. The social control measure asked whether neighbours would take action (from 1 "strongly agree" to 4 "strongly disagree") if: children were skipping school or loitering, spray-painting graffiti on a building, or showing disrespect to an adult; or if a fight broke out in front of their house, or the fire station closest to home was threatened by budget cuts. Social cohesion assessed respondents' level of agreement (from 1 "strongly agree" to 4 "strongly disagree") that people in their neighborhood generally don't get along, do not share the same values, can be trusted, are willing to help their neighbours, or are close-knit (last three items reverse coded). Items were summed with higher scores indicating greater collective efficacy.

\section{Pathway variables}

Maternal health behaviours

Maternal smoking and breastfeeding behaviours were obtained in wave 3 . These behaviours may be disrupted through violence exposure [26, 27] and are associated with childhood asthma/wheeze risk. 


\section{Statistical analyses}

Differences in means or proportions between asthmatics and nonasthmatics were evaluated using Chi-squared or unpaired t-tests, as appropriate. The correlation between continuous variables was examined, given the concern for collinearity.

These multilevel data included individuals at level 1 nested within $80 \mathrm{NCs}$ at level 2. To account for potential neighbourhood variation in asthma, we used multilevel logistic regression models [34], estimated using MLwiN, version 2.10 (University of Bristol, Bristol, UK), building models in a stepwise fashion [35].

As represented in figure 2, we examined potential pathways linking community violence, as well as confounders of these associations. First, we modelled the log odds of asthma as a function of neighbourhood violence, adjusting for the child's age, sex, race/ethnicity and maternal asthma, conditional on the random effect for neighbourhoods. We then introduced individual-level confounders: socioeconomic covariates (caregiver's education and equivalised household income) (model 2); family violence exposure in the home (model 3); mother's smoking status and breastfeeding history (model 4). The final model (model 5) adjusted for neighbourhood-level confounding (concentrated disadvantage, collective efficacy, and social disorder at level 2).

\section{Sensitivity analysis}

Sensitivity analyses considered alternative asthma indicators (current asthma and unconfirmed possible asthma).

\section{RESULTS}

Sample characteristics are summarised in table 2. Overall, 19\% of the children had diagnosed asthma. A greater proportion of those exposed to medium (35.2\%) or high (43\%) community violence had asthma, compared with those with low ETV $(21.8 \%)$ ( $p<0.01$ for both). More African Americans, relative to Whites or Mexicans, had asthma $(25.8$ versus $17.3 \%(p<0.004)$ and 25.8 versus $12.1 \%(\mathrm{p}<0.001)$, respectively). Fewer Mexicans had asthma than Whites (12.1 versus $17.3 \%$; $<<0.03)$ ) or nonMexican Hispanics (12.1 versus $21.5 \%$; $<<0.001)$ ). More males $(22.6 \%)$ than females $(15.4 \%)$ had asthma $(\mathrm{p}<0.001)$. Children of mothers with asthma were more likely to have asthma compared to those without a maternal history (43.1 versus $15.1 \% ; \mathrm{p}<0.0001)$ ). Asthma was also higher among children exposed to high $(21 \% ; \mathrm{p}<0.01)$ or low $(19 \% ; \mathrm{p}<0.03)$ family violence, compared with no family violence (13.2\%). Asthma was increased in areas of high concentrated disadvantage $(23.2 \%)$ compared to medium $(16.4 \%$; $<<0.01)$ or low $(17.3 \%$; $\mathrm{p}<0.01)$.

Experiencing violence in the neighbourhood was significantly related to family violence in the home, the child's age, equivalised income and the neighbourhood constructs, although the correlations were weak, minimising concerns for collinearity (table 3). Equivalised income, neighbourhood disadvantage, social disorder and collective efficacy were more moderately correlated. However, adding these covariates to the models in various combinations did not significantly influence the $\beta$ estimates or standard errors, again lessening concern for collinearity (data not shown) [36].
In hierarchical models, community violence, when measured continuously, was significantly associated with elevated asthma risk. Unadjusted analyses revealed an almost 50\% increased risk in asthma for every unit increase in the ETV score (OR 1.47, 95\% CI 1.30-1.68). The effects persisted after adjusting for all individual- and neighbourhood-level covariates (OR 1.28, 95\% CI 1.09-1.49). Results were robust to alternative asthma definitions (current asthma and unconfirmed possible asthma) (data not shown).

The odds ratio for asthma by exposure to community violence (measured in tertiles) is presented in table 4. Model 1 is adjusted for maternal asthma, child race and sex. In this model, exposure to medium (OR 1.55, 95\% CI 1.14-2.11) and high (OR 1.53 , 95\% CI 1.11-2.10) levels of community violence was associated with a similarly elevated risk of asthma compared to those with low exposure. The effects for community violence were essentially unchanged after further adjusting for all other individual- and neighbourhood-level confounders (model 5).

In the fully adjusted model 5, male sex and maternal asthma were independently associated with increased asthma risk. Neighbourhood-level constructs were not significantly related to asthma.

Notably, African American race remained independently associated with increased asthma risk in models including community violence and all other individual-level covariates (model 4). Further adjusting for all three neighbourhood constructs simultaneously attenuated this effect (model 5). In order to ascertain which construct was responsible for the attenuation we added variables one at a time. Further adjustment for concentrated disadvantage and social disorder added to Model 4 (one at a time) did not significantly change the observed relationship with African American race (OR 1.54, 95\% CI 1.02-2.3, and OR 1.62, 95\% CI 1.02-2.6, respectively). Upon further adjusting model 4 for collective efficacy, the elevated risk in African Americans remained, albeit at borderline significance (OR 1.49, 95\% CI 0.99-2.24) similar to the effect estimate in model 5 (including all three neighbourhood constructs). This suggested that reduced levels of collective efficacy, in part, explains the persistent increased risk for asthma seen in these African American children.

\section{DISCUSSION}

To our knowledge, this is the first longitudinal, multilevel study to demonstrate a significant association between community violence exposure and increased risk for asthma development in urban children. This association was robust to controlling for important individual-level factors (race/ ethnicity, SES, maternal health behaviours and family violence), and neighbourhood-level confounders (concentrated disadvantage, social disorder and collective efficacy), and was consistent across alternative outcomes (e.g. current asthma or undiagnosed possible asthma).

Our findings contribute to a growing body of evidence linking community violence to asthma expression [10, 11, 13]. A number of mechanisms underlying this association have been proposed. Violence exposure has been conceptualised as a psychological and environmental stress that taxes both individuals and communities [5]. At the individual level, increased stress may lead to the dysregulation of the 
TABLE 2 Distribution of covariates across the primary asthma outcome ${ }^{\#}$

\begin{tabular}{|c|c|c|c|c|}
\hline & All & Asthma & No asthma & p-value \\
\hline Cases & 2071 & $395(19)$ & 1676 (81) & \\
\hline Low & $693(33.5)$ & $86(21.8)$ & 607 (36.2) & \\
\hline Medium & $672(32.4)$ & $139(35.2)$ & $533(31.8)$ & \\
\hline High & $706(34.1)$ & $170(43.0)$ & $536(32.0)$ & \\
\hline Low violence exposure & $783(37.8)$ & $149(37.7)$ & $634(37.8)$ & \\
\hline High violence exposure & $767(37.0)$ & $161(40.8)$ & $606(36.2)$ & \\
\hline Missing & $279(13.5)$ & $53(13.4)$ & $226(13.5)$ & \\
\hline Child age & $2071 \pm 10.5$ & $395 \pm 10.7$ & $1676 \pm 10.5$ & 0.26 \\
\hline Child race & & & & $<0.0001$ \\
\hline White & $300(14.5)$ & $52(13.2)$ & $248(14.8)$ & \\
\hline Child's sex & & & & $<0.0001$ \\
\hline Female & $1018(49.2)$ & $157(39.8)$ & $861(51.4)$ & \\
\hline Male & $1053(50.8)$ & $238(60.3)$ & $815(48.6)$ & \\
\hline Caregiver's education & & & & 0.001 \\
\hline Less than high school & $816(39.4)$ & $138(34.9)$ & $678(40.5)$ & \\
\hline High school degree & $279(13.5)$ & $38(9.6)$ & $241(14.4)$ & \\
\hline Some university or higher & $936(45.2)$ & $213(53.9)$ & $723(43.1)$ & \\
\hline Missing & $40(1.9)$ & $6(1.5)$ & $34(2.0)$ & \\
\hline Equivalised income US\$ & & & & 0.20 \\
\hline$<20,000$ & $462(22.3)$ & $93(23.5)$ & 369 (22.0) & \\
\hline$>20,000$ & $1491(72.0)$ & $273(69.1)$ & $1218(72.7)$ & \\
\hline Current & $501(24.2)$ & $100(25.3)$ & $401(23.9)$ & \\
\hline Missing & $19(0.9)$ & $5(1.3)$ & $14(0.8)$ & \\
\hline Breastfeeding status & & & & 0.45 \\
\hline Not breastfed & $1078(52.1)$ & $216(54.7)$ & $862(51.4)$ & \\
\hline Breastfed & $959(46.3)$ & $174(44.1)$ & $785(46.8)$ & \\
\hline Missing & $34(1.6)$ & $5(1.3)$ & $29(1.7)$ & \\
\hline Concentrated disadvantage & & & & 0.002 \\
\hline Low & $612(29.6)$ & $106(26.8)$ & $506(30.2)$ & \\
\hline Medium & $725(35.0)$ & $119(30.1)$ & $606(36.2)$ & \\
\hline High & $734(35.4)$ & $170(43.0)$ & $564(33.7)$ & \\
\hline Collective efficacy & & & & 0.25 \\
\hline Low & $697(34.0)$ & $127(32.15)$ & $570(34.0)$ & \\
\hline Medium & $775(37.0)$ & $162(41.01)$ & $613(36.6)$ & \\
\hline High & $599(29.0)$ & $106(26.84)$ & $493(29.4)$ & \\
\hline Social disorder & & & & 0.80 \\
\hline Low & $600(0.29)$ & 109 (27.59) & 491 (29.3) & \\
\hline Medium & $695(0.34)$ & $135(34.18)$ & $560(33.4)$ & \\
\hline High & $776(0.37)$ & 151 (38.23) & 625 (37.3) & \\
\hline
\end{tabular}

Data are presented as $n, n(\%)$ or mean $\pm \mathrm{SD}$, unless otherwise stated. ${ }^{*}$ : childhood asthma outcome is defined as having ever been diagnosed with asthma or ever taking prescription asthma medicine; ": for differences between nonmissing multigroup comparisons using Chi-squared distribution. 


\begin{tabular}{|c|c|c|c|c|c|c|c|}
\hline 1) Community violence & $1.00(0.00)$ & & & & & & \\
\hline 2) Child age & $0.16(0.00)$ & 1.00 & & & & & \\
\hline 4) Violence in family & $0.17(0.00)$ & $-0.02(0.33)$ & $-0.15(0.00)$ & 1.00 & & & \\
\hline 5) Concentrated disadvantage & $0.34(0.00)$ & $0.03(0.19)$ & $-0.35(0.00)$ & $0.16(0.00)$ & 1.00 & & \\
\hline 6) Social disorder & $0.11(0.00)$ & $0.02(0.29)$ & $-0.46(0.00)$ & $0.13(0.00)$ & $0.56(0.00)$ & 1.00 & \\
\hline 7) Collective efficacy & $-0.06(0.00)$ & $-0.02(0.30)$ & $0.38(0.00)$ & $-0.10(0.00)$ & $-0.38(0.00)$ & $-0.73(0.00)$ & 1.00 \\
\hline
\end{tabular}

hypothalamic-pituitary-adrenal (HPA) and sympathetic-adrenal-medullary (SAM) axes, disrupting immune and respiratory processes, and producing an increased risk of inflammatory diseases, such as asthma [3, 5]. Indeed, we previously documented psychopathology and cortisol dysregulation in school-aged children exposed to higher levels of community violence in neighbourhoods in Boston, MA, USA [8]. The child's primary caregiver (primarily the mother) obviously share the violent environment, and thus violence may operate through influencing behaviours and functioning in the mother that then impact asthma expression in the child. For example, violence exposure may result in increased smoking in mothers, thus increasing the child's exposure [26]. Similarly, low-income females living with violence may be less likely to breastfeed [27], which may enhance asthma risk. However, adjusting for maternal smoking and breastfeeding behaviours did not reduce the observed effect. Community violence exposure may also result in poor psychological functioning in mothers (e.g. anxiety and depression) [37] that may, in turn, disrupt parenting behaviours and lead to greater stress for the child and altered emotional development [38]. Other studies show that children exposed to violence tend to express higher levels of negative emotion (e.g. anxiety and depression). More harmful stress reactivity and negative emotion, in turn, have been linked to asthma [38]. Additionally, concern about safety may cause parents to restrict their children's outdoor activity and close windows, potentially increasing exposure to indoor pollutants. Children kept indoors are also more likely to be sedentary; this, in turn, may be linked to obesity, another risk factor for asthma [5]. Finally, violence-induced stress may potentiate asthma-inducing effects of other environmental pollutants (e.g. air pollution) [3, 12]. Future work should examine these potential pathways more directly.

In models including community violence, concentrated disadvantage and social disorder, as well as individual-level covariates, an increased risk of asthma in African American children remained. Notably, when accounting for collective efficacy at the neighbourhood level, the elevated risk in African Americans was no longer significant. This finding is corroborated by a Chicago study showing lower prevalence of a composite of respiratory problems (e.g. asthma, emphysema and other breathing problems) in association with increased collective efficacy in adults [39]. As pointed out by CAGNEY and
BROWNING [39], mechanisms may include: 1) social control of adverse health behaviours (e.g. smoking); 2) access to highquality healthcare promoting advantageous health practices (e.g. breastfeeding); 3) empowerment to act collectively to address adverse environmental factors (e.g. polluting traffic sources, housing conditions); and 4) less social isolation $[40,41]$. These pathways should be examined empirically in future work.

\section{Strengths and limitations}

Strengths of the study include the use of longitudinal data, a large urban sample, application of item response theory to summarise community violence exposure, control for other forms of violence (i.e. family violence) as well as other important confounders, and the employment of multilevel analyses to, at least in part, control for unmeasured neighbourhood factors. Moreover, findings were robust to alternative specifications of the asthma/wheeze outcome. Results should also be interpreted in light of study limitations. We cannot rule out some unmeasured confounding factor that is correlated both with community violence and with asthma risk. Exposure to community violence is just one type of stressor experienced by children, particularly those living in lower-income urban environments. While we adjust for other forms of violence (i.e. family violence in the home), we did not have information on other stressors (e.g. other negative life events, racism and housing stress) in this dataset. Violence exposure was ascertained through questionnaires only; there were no available biomarkers of potential stress pathways operating between community violence and asthma (e.g. cortisol disruption) in the PHDCN sample. Those experiencing higher levels of community violence may also be exposed to poorer quality housing and greater traffic, or other polluting sources. Although we considered neighborhood disadvantage to partly account for this, the study did not include questions on household characteristics (e.g. allergens and housing quality) or more direct measures of the physical toxins that may also disproportionately burden residents in more disadvantaged communities [17]. We do have an ongoing birth cohort study examining risks for childhood asthma in Boston that is directly measuring indoor allergens and indoor/outdoor air pollution, violence exposure measures and other relevant stressors, as well as biomarkers, that will be able to address these limitations in the future [42]. Finally, the analysis was limited to Chicago and may not be generalisable beyond this region. 
TABLE 4 Associations between community violence exposure and childhood asthma: hierarchical logistic regression\#

\begin{tabular}{|c|c|c|c|c|c|}
\hline & Model 1 & Model 2 & Model 3 & Model 4 & Model 5 \\
\hline \multicolumn{6}{|l|}{ Community violence } \\
\hline Low & 1.00 & 1.00 & 1.00 & 1.00 & 1.00 \\
\hline High & $1.53(1.11-2.10)$ & $1.52(1.11-2.10)$ & $1.55(1.12-2.15)$ & $1.56(1.12-2.16)$ & $1.56(1.12-2.18)$ \\
\hline \multicolumn{6}{|l|}{ Child's Age } \\
\hline Centred age at Wave 3 & $1.01(0.97-1.06)$ & $1.01(0.97-1.07)$ & $1.01(0.96-1.06)$ & $1.01(0.96-1.06)$ & $1.01(0.96-1.06)$ \\
\hline Males & $1.70(1.35-2.15)$ & $1.69(1.34-2.14)$ & $1.69(1.33-2.14)$ & $1.70(1.34-2.15)$ & $1.70(1.34-2.16)$ \\
\hline \multicolumn{6}{|l|}{ Child's race/ethnicity } \\
\hline White & 1.00 & 1.00 & 1.00 & 1.00 & 1.00 \\
\hline African American & $1.43(1.00-2.07)$ & $1.52(1.03-2.24)$ & $1.55(1.05-2.29)$ & $1.61(1.08-2.39)$ & $1.57(0.98-2.52)$ \\
\hline Mexican & $0.70(0.47-1.03)$ & $0.80(0.52-1.23)$ & $0.79(0.51-1.23)$ & $0.80(0.51-1.26)$ & $0.75(0.47-1.20)$ \\
\hline Ever & $3.97(3.02-5.24)$ & $3.96(3.00-5.23)$ & $4.01(3.03-5.31)$ & $4.01(3.03-5.31)$ & $4.02(3.04-5.32)$ \\
\hline Missing & $1.07(0.52-2.20)$ & $1.07(0.52-2.19)$ & $1.05(0.47-2.35)$ & $1.05(0.47-2.35)$ & $1.03(0.46-2.32)$ \\
\hline \multicolumn{6}{|l|}{ Household income US\$ } \\
\hline$>20,000$ & & 1.00 & 1.00 & 1.00 & 1.00 \\
\hline$<20,000$ & & $0.93(0.69-1.27)$ & $0.94(0.69-1.28)$ & $0.96(0.70-1.32)$ & $0.92(0.66-1.27)$ \\
\hline Missing & & $1.24(0.73-2.12)$ & $1.25(0.73-2.13)$ & $1.29(0.75-2.21)$ & $1.26(0.73-2.18)$ \\
\hline \multicolumn{6}{|l|}{ Maternal Education } \\
\hline University education & & 1.00 & 1.00 & 1.00 & 1.00 \\
\hline Less than high school & & $0.81(0.61-1.08)$ & $0.83(0.62-1.10)$ & $0.84(0.62-1.12)$ & $0.83(0.62-1.11)$ \\
\hline High school graduate & & $0.59(0.39-0.87)$ & $0.59(0.40-0.88)$ & $0.60(0.40-0.90)$ & $0.59(0.39-0.88)$ \\
\hline Missing & & $0.78(0.31-1.95)$ & $0.78(0.31-1.96)$ & $0.80(0.32-2.00)$ & $0.81(0.32-2.04)$ \\
\hline \multicolumn{6}{|l|}{ Maternal smoking } \\
\hline Current & & & & $0.90(0.67-1.20)$ & $0.91(0.68-1.22)$ \\
\hline Missing & & & & $1.75(0.56-5.49)$ & $1.71(0.54-5.40)$ \\
\hline \multicolumn{6}{|l|}{ Breastfeeding } \\
\hline Never & & & & 1.00 & 1.00 \\
\hline Ever & & & & $1.03(0.81-1.33)$ & $1.02(0.79-1.31)$ \\
\hline Missing & & & & $0.69(0.23-2.03)$ & $0.69(0.24-2.05)$ \\
\hline \multicolumn{6}{|c|}{ Concentrated disadvantage } \\
\hline Low & & & & & 1.00 \\
\hline Medium & & & & & $0.8(50.57-1.26)$ \\
\hline High & & & & & $0.90(0.57-1.41)$ \\
\hline \multicolumn{6}{|l|}{ Collective efficacy } \\
\hline Low & & & & & 1.00 \\
\hline Medium & & & & & $0.94(0.69-1.29)$ \\
\hline High & & & & & $0.73(0.46-1.15)$ \\
\hline \multicolumn{6}{|l|}{ Social disorder } \\
\hline Low & & & & & 1.00 \\
\hline Medium & & & & & $1.14(0.77-1.69)$ \\
\hline High & & & & & $0.95(0.59-1.54)$ \\
\hline
\end{tabular}

Data are presented as odds ratio (95\% confidence interval). CTS: conflict tactics scale. ${ }^{\#}: n=2,071 ; "$ : equivalised income was calculated by dividing household income by the square root of the number of household members. 
Our findings underscore the potential role of community violence in explaining urban asthma risk in the USA. From a policy perspective, our findings suggest that public health interventions outside of the biomedical model (e.g. neighbourhood safety programmes) may be advantageous in reducing the asthma burden in disadvantaged populations. Research to more fully elucidate the excess asthma burden among African American children, and the potential role of collective efficacy in reducing that burden, is also warranted. These analyses begin to address the recognised need to consider a shared conceptual framework considering social, physical and behavioural factors, integrating multilevel methods to elucidate the complexities of asthma disparities [42, 43].

\section{SUPPORT STATEMENT}

The Project on Human Development in Chicago Neighborhoods was supported by grants from the National Institute of Justice, the National Institute of Mental Health and the John D.and Catherine T. MacArthur Foundation. Collection of the asthma outcomes data was, in part, funded through K08 HL04187 (R.J. Wright). During preparation of the manuscript, R.J. Wright was funded by R01 HL080674, M.J. Sternthal by grant T32-ES07069-29, and M.J. Sternthal by the Leaves of Grass Foundation.

\section{STATEMENT OF INTEREST}

None declared.

\section{REFERENCES}

1 Cesaroni G, Farchi S, Davoli M, et al. Individual and area-based indicators of socioeconomic status and childhood asthma. Eur Respir J 2003; 22: 619-624.

2 Wright RJ, Subramanian SV. Advancing a multilevel framework for epidemiologic research on asthma disparities. Chest 2007; 132: 757S-769S.

3 Wright RJ, Cohen RT, Cohen S. The impact of stress on the development and expression of atopy. Curr Opin Allergy Clin Immunol 2005; 5: 23-29.

4 Wright RJ. Moving towards making social toxins mainstream in children's environmental health. Curr Opin Pediatr 2009; 21: 222-229.

5 Wright RJ. Health effects of socially toxic neighborhoods: the violence and urban asthma paradigm. Clin Chest Med 2006; 27: 413-421.

6 Sheehan K, DiCara JA, LeBailly S, et al. Children's exposure to violence in an urban setting. Arch Pediatr Adolesc Med 1997; 151: 502-504.

7 Suglia SF, Ryan L, Wright RJ. Creation of a community violence exposure scale: accounting for what, who, where, and how often. J Trauma Stress 2008; 21: 479-486.

8 Suglia SF, Staudenmayer J, Cohen S, et al. Posttraumatic stress symptoms related to community violence and children's diurnal cortisol response in an urban community-dwelling sample. Int $J$ Behav Med 2010; 17: 43-50.

9 Murali R, Chen E. Exposure to violence and cardiovascular and neuroendocrine measures in adolescents. Ann Behav Med 2005; 30: 155-163.

10 Swahn MH, Bossarte RM. The associations between victimization, feeling unsafe, and asthma episodes among US high-school students. Am J Public Health 2006; 96: 802-804.

11 Wright RJ, Mitchell H, Visness CM, et al. Community violence and asthma morbidity: the Inner-City Asthma Study. Am J Public Health 2004; 94: 625-632.
12 Clougherty JE, Levy JI, Kubzansky LD, et al. Synergistic effects of traffic-related air pollution and exposure to violence on urban asthma etiology. Environ Health Perspect 2007; 115: 1140-1146.

13 Berz JB, Carter AS, Wagmiller RL, et al. Prevalence and correlates of early onset asthma and wheezing in a healthy birth cohort of 2to 3-year olds. J Pediatr Psychol 2007; 32: 154-166.

14 Suglia FS, Bosquet Enlow M, Kullowatz A, et al. Maternal intimate partner violence predicts increased asthma incidence in children: buffering effects of supportive caregiving. Arch Pediatr Adolesc Med 2009; 163: 244-250.

15 Saegert S, Winkel G. Crime, social capital, and community participation. Am J Community Psychol 2004; 34: 219-233.

16 Fisher EB, Strunk RC, Sussman LK, et al. Community organization to reduce the need for acute care for asthma among African American children in low-income neighborhoods: the Neighborhood Asthma Coalition. Pediatrics 2004; 114: 116-123.

17 O'Neill MS, Jerrett M, Kawachi I, et al. Health, wealth, and air pollution: advancing theory and methods. Environ Health Perspect 2003; 111: 1861-1870.

18 Sampson RJ, Raudenbush SW, Earls F. Neighborhoods and violent crime: a multilevel study of collective efficacy. Science 1997; 277: 918-924.

19 Bingenheimer JB, Brennan RT, Earls FJ. Firearm violence exposure and serious violent behavior. Science 2005; 308: 1323-1326.

20 Brennan RT, Molnar BE, Earls F. Refining the measurement of exposure to violence (ETV) in urban youth. J Community Psychol 2007; 35: 603-618.

21 Selner-O'Hagan MB, Kindlon DJ, Buka SL, et al. Assessing exposure to violence in urban youth. J Child Psychol Psychiatry 1998; 39: 215-224.

22 Ferris BGJ. Epidemiology standardization project (American Thoracic Society). Am Rev Respir Dis 1978; 118: 7-53.

23 Jenkins MA, Clarke JR, Carlin JB, et al. Validation of questionnaire and bronchial hyperresponsiveness against respiratory physician assessment in the diagnosis of asthma. Int J Epidemiol 1996; 25: 609-618.

24 Flores G, Tomany-Korman SC. Racial and ethnic disparities in medical and dental health, access to care, and the use of services among Hispanics in the United States. Pediatrics 2008; 18: 93-97.

25 Zwarenstein M, Bheekie A, Lombard C, et al. Educational outreach to general practitioners reduces children's asthma symptoms: a cluster randomised controlled trial. Implement Sci 2007; 2: 30.

26 Ganz ML. The relationship between external threats and smoking in central Harlem. Am J Public Health 2000; 90: 367-371.

27 Chin NP, Solomonik A. Inadequate: a metaphor for the lives of low-income women? Breastfeed Med 2009; 4: S41-S43.

28 Buhmann B, Rainwater L, Schmaus G, et al. Equivalence scales, well-being, inequality, and poverty: Sensitivity estimates across ten countries using the Luxembourg Income Study (LIS) database. Rev Income Wealth 1988; 34: 115-142.

29 Straus MA. The Conflict Tactics Scales and its critics: an evaluation and new data on validity and reliability. In: Straus MA, Gelles RJ, eds. Physical Violence in American Families: Risk Factors and Adaptations to Violence in 8,145 Families. Piscataway, Transaction Publishers; 1990. pp. 49-73.

30 Connelly CD, Newton RR, Aarons GA. A psychometric examination of English and Spanish versions of the Revised Conflict Tactics scales. J Interpers Violence 2005; 20: 1560-1579.

31 Sampson RJ, Morenoff JD, Felton E. Beyond social capital: spatial dynamics of collective efficacy for children. Am Sociol Rev 1999; 64: 633-660.

32 Markowitz FE, Paul E B, Allen EL, et al. Extending social disorganizaion theory: modeling the relationship between cohesion, disorder, and fear. Criminology 2001; 39: 293-319.

33 Browning CR, Cagney KA. Neighborhood structural disadvantage, collective efficacy, and self-rated physical health in an urban setting. J Health Soc Behav 2002; 43: 383-399. 
34 Raudenbush S, Bryk A. Hierarchical Linear Models: Applications and Data Analysis Methods. Thousand Oaks, Sage Publications, 2002.

35 Rasbash J, Steele F, Browne W, et al. A User's Guide to MLwiN Version 2.10. London, Centre for Multilevel Modelling, Institute of Education; 2008.

36 Hosmer DW, Lemeshow S. Applied Logistic Regression. New York, John Wiley \& Sons, 1989.

37 Clark C, Ryan L, Kawachi I, et al. Witnessing community violence in residential neighborhoods: a mental health hazard for urban women. J Urban Health 2007; 85: 22-38.

38 Wright R, Bosquet EM. Maternal stress and perinatal programming in the expression of atopy. Exp Rev Clin Immun 2008; 4 $535-538$
39 Cagney KA, Browning CR. Exploring neighborhood-level variation in asthma and other respiratory diseases. J Gen Intern Med 2004; 19: 229-236.

40 Ahern J, Galea S, Hubbard A, et al. Neighborhood smoking norms modify the relation between collective efficacy and smoking behavior. Drug Alcohol Depend 2009; 100: 138-145.

41 Fullilove MT. Promoting social cohesion to improve health. J Am Med Womens Assoc 1998; 53: 72-76.

42 Wright RJ, Suglia SF, Levy J, et al. Transdisciplinary research strategies for understanding socially patterned disease: the Asthma Coalition on Community, Environment, and Social Stress (ACCESS) project as a case study. Cien Saude Colet 2008; 13: 1729-1742.

43 Cruz AA, Bateman ED, Bousquet J. The social determinants of asthma. Eur Respir J 2010; 35: 239-242. 